Atualização / Update

\title{
Transplante de célula-tronco hematopoética para síndrome mielodisplásica
}

\section{Bone marrow transplantation in myelodysplastic syndromes}

Daniel G. Tabak

Simone C. M. Pereira ${ }^{2}$

Marta C. Nogueira ${ }^{3}$

\begin{abstract}
As síndromes mielodisplásicas (SMD) constituem um grupo de doenças hematológicas caracterizadas por citopenias crônicas, associadas a uma maturação celular anormal. A melhor forma de classificação atual destas patologias é o International Prognostic Scoring System (IPSS), que se baseia no grau de citopenia, número de mieloblastos na medula óssea e alterações citogenéticas. Há quatro estágios: baixo risco, riscos intermediário-1 e 2 e alto risco. Um grupo destes pacientes pode ser curado com o transplante de células-tronco hematopoéticas (TCTH). Esta forma de tratamento pode ser considerada para pacientes com idade inferior a 60 anos, que possuam um doador familiar HLA-idêntico. A opção por esta modalidade terapêutica depende de alguns critérios, que incluem o IPSS, o risco de progressão de doença, o risco de infecção e o estado geral do paciente. O TCTH autólogo pode ser considerado em pacientes que alcancem uma remissão completa citogenética e que não disponham de doador HLAidêntico. Em pacientes não candidatos ao TCTH mieloablativo, uma possibilidade éo transplante com regimes de intensidade reduzida. Estudos recentes têm demonstrado resultados favoráveis com esta opção terapêutica, pois, apesar do alto rico de recaída, as taxas de mortalidade associada ao procedimento são menores. Os pacientes com SMD devem ser dispostos em ensaios clínicos que considerem as comorbidades, DECH e riscos de recaída. Rev. Bras. Hematol. Hemoter. 2010;32(Supl. 1):66-70.
\end{abstract}

Palavras-chave: Transplante de células-tronco hematopoéticas; síndromes mielodisplásicas.

\section{Introdução}

As síndromes mielodisplásicas (SMDs) correspondem a um distúrbio clonal da célula-tronco hematopoética, podendo ter várias formas de apresentação, variando desde aplasia de medula até estágios pré-leucêmicos. Elas abrangem uma série de condições hematológicas caracterizadas por citopenias crônicas (anemia, neutropenia e trombocitopenia) acompanhadas de maturação celular anormal. A doença é frequentemente progressiva, com evolução para leucemia mieloide aguda em cerca de $30 \%$ dos pacientes. O transplante de células-tronco hematopoéticas (TCTH) é usualmente considerado o tratamento de escolha para pacientes jovens com doador aparentado, e muitos autores consideram o transplante de medula óssea (TMO) como a única modalidade terapêutica curativa. Embora ocorra na infância, a maioria dos pacientes é idosa, com menos de $25 \%$ com idade inferior a 65 anos.

\section{Prognóstico / Estratificação de risco}

Atualmente, a melhor forma de estratificação das SMDs é o IPSS (International Prognostic Scoring System), que divide as SMDs em quatro grupos, de acordo com o número de citopenias, percentual de mieloblastos na medula óssea e anomalias citogenéticas.

\footnotetext{
${ }^{1}$ Hematologista/Oncologista. Médico da Terapia Celular da Clínica São Vicente da Gávea - Rio de Janeiro-RJ.

${ }_{3}^{2}$ Médica do ambulatorio de pré-TMO do Cemo/Inca - Rio de Janeiro-RJ.

${ }^{3}$ Hematologista e hemoterapeuta. Chefe da unidade de pacientes internos do Cemo/Inca - Rio de Janeiro-RJ.
}

Terapia Celular da Clínica São Vicente da Gávea - Rio de Janeiro-RJ. 
Apesar do IPSS não conseguir identificar, dentre os pacientes com baixo risco (IPSS baixo ou intermediário-1), os de pior prognóstico, é um bom escore para aqueles com alto risco (IPSS alto risco e intermediário-2). ${ }^{1}$ Baseando-se no IPSS, os pacientes de risco alto ou intermediário-2 têm indicação de tratamento agressivo, como o TCTH, visto o alto risco de evolução para leucemias, má resposta aos tratamentos convencionais e alta mortalidade.

Em uma análise retrospectiva, os pacientes classificados como baixo risco ou risco intermediário-2 foram beneficiados pelo transplante de medula óssea (TMO) tardiamente, porém antes da transformação para leucemia. ${ }^{2}$

\section{Transplante de células-tronco hematopoéticas autólogo nas síndromes mielodisplásicas}

As principais restrições ao uso do transplante de células-tronco hematopoéticas autólogo em pacientes com SMD se devem às dificuldades em se alcançarem remissões citogenéticas e na dificuldade de mobilização de precursores hematopoéticos visto os tratamentos anteriores. ${ }^{3}$ De Witte e cols. ${ }^{4}$ publicaram o primeiro estudo prospectivo no sentido de avaliar o papel da consolidação intensiva com transplante de precursores hematopoéticos em pacientes portadores de SMD que obtiveram remissão completa após quimioterapia intensiva. Cem de 184 pacientes foram avaliados em remissão hematológica completa após um ou dois ciclos de quimioterapia com idarrubicina, citarabina e etoposido. A morte relacionada ao tratamento foi observada em 25 pacientes, e 55 pacientes demonstraram persistência da doença ou medula hipoplásica. Os resultados foram analisados por intenção de tratar e não propriamente pelo tratamento recebido. Dos 39 pacientes que dispunham de um doador HLA idêntico familiar, 26 (67\%) foram transplantados. Apenas 35 dos 61 pacientes $(57 \%)$ destinados a receber intensificação com o transplante autogênico em primeira remissão hematológica foram assim realmente tratados. A sobrevida livre de eventos (SLE), em quatro anos, foi semelhante para os dois grupos ( $31 \%$ alogênico x $27 \%$ autogênico), porém, deve ser analisada no contexto dos vários fatores que limitam a utilização desta estratégia para pacientes portadores de SMD. ${ }^{4}$

\section{Seleção do doador}

Para os pacientes com IPSS alto, Int-2 ou Int-1 e baixo risco em estádios avançados, na ausência de um doador familiar HLA idêntico, faz-se necessário a busca por doadores não aparentados, apesar da maior probabilidade de complicações após o TCTH (maior toxicidade relacionada ao procedimento e maior risco de doença-enxerto-contra-hospedeiro - DECH aguda grave). ${ }^{5} \mathrm{Na}$ tentativa de minimizar estas complicações, esses autores concluíram que, para transplantes não aparentados com compatibilidade 10/10, a sobrevida após o transplante em fases mais avançadas era semelhante à sobrevida dos transplantes com doadores aparentados. Entretanto, observou-se uma maior incidência de DECH grave com o uso de doadores não aparentados.

\section{Papel do transplante de células-tronco hematopoéticas com doador não aparentado}

Vários estudos vêm demonstrando que o TCTH não aparentado pode ser uma estratégia curativa para pacientes com SMD. O maior estudo sobre TCTH de doadores não aparentados é um relato de 510 pacientes que foram transplantados no período de 1988 a 1998, com mediana de idade de 38 anos. Dos 437 pacientes avaliados, a probabilidade de sobrevida livre de doença (SLD) em dois anos foi de 29\%, e a mortalidade relacionada ao tratamento (TRM) foi de 54\%, tendo sido associada à idade mais avançada do doador e do receptor, disparidade HLA e positividade para citomegalovírus (CMV) no receptor. Foi observada uma tendência mais favorável com regimes que não utilizaram a radioterapia corporal total. ${ }^{6}$ De Witte e col ${ }^{7}$ relataram os resultados de 198 pacientes submetidos a TCTH de doador não aparentado. A taxa de mortalidade relacionada ao procedimento foi de $58 \%$ e a recidiva de $41 \%$.

\section{Transplante de células de cordão umbilical}

A utilização de precursores hematopoéticos HLA idênticos de um doador familiar e mesmo de um doador não consanguíneo com um ou dois antígenos distintos é considerada hoje uma alternativa adequada ao uso de medula óssea como fonte de precursores hematopoéticos para transplante em pacientes pediátricos e adultos. $\mathrm{O}$ Eurocord relatou a experiência com cinquenta pacientes com seguimento mediano de 21 meses e uma sobrevida livre de progressão de 30\%. A utilização crescente dos transplantes duplos de células de cordão umbilical nos permite visualizar sua maior aplicabilidade no tratamento da SMD. ${ }^{8}$

\section{Idade}

Vários estudos demonstram um aumento da frequência e gravidade de complicações pós-transplante com a idade. Esta é uma preocupação real em portadores de SMD, uma vez que a idade mediana ao diagnóstico é de 70 anos. ${ }^{1}$ Deeg e cols. ${ }^{9}$ publicaram a experiência de Seattle com cinquenta pacientes portadores de SMD submetidos a transplante alogênico. A idade variou de 55 a 66 anos e apenas um paciente era considerado de baixo risco pelo IPSS. Os regimes de condicionamento foram: ciclofosfamida associada à irradiação corporal total em 19 pacientes, 7 pacientes receberam doses reduzidas de bussulfano também associadas à radioterapia e 27 receberam bussulfano na dose de $16 \mathrm{mg} / \mathrm{kg}$ associado à ciclofosfamida. Este grupo incluiu 16 pacientes nos quais a dose de bussulfano foi planejada para produzir um nível sérico 
entre 600 e $900 \mathrm{ng} / \mathrm{ml}$. Trinta e quatro pacientes receberam o enxerto de um irmão HLA idêntico. Entre 13 pacientes portadores de anemia refratária (AR), a estimativa de sobrevida em três anos foi de 59\%. A sobrevida livre de eventos apresentou uma correlação inversa com a classificação de risco citogenético e com o IPSS. Curiosamente, a sobrevida em todos os grupos foi superior nos pacientes que receberam doses ajustadas de bussulfano, indicando também um potencial para a utilização de regimes não mieloablativos nestes indivíduos. Desta forma, a idade não constitui uma contraindicação absoluta para pacientes selecionados portadores de SMD.

Os regimes de intensidade reduzida (RIST), além de promoverem o efeito enxerto-contra-tumor, permitem a realização do TMO para pacientes entre 70 e 75 anos, ou pacientes jovens com comorbidades. ${ }^{10,11}$
Na população pediátrica, o TCTH é considerado o tratamento de escolha, com bons resultados principalmente quando da utilização de doadores aparentados. ${ }^{12,13} \mathrm{O}$ grupo de Seattle, em um estudo retrospectivo, analisou 94 crianças, e, entre esses pacientes, a estimativa de sobrevida em três anos foi de 50\%, com uma sobrevida livre de eventos de $41 \% .^{14}$

\section{Recomendação de tratamento}

O NCCN ( National Comprehensive Cancer Network) ${ }^{15}$ sugere que a escolha do tratamento deve se basear em três características clínicas: idade do paciente, performance status e IPSS. A nossa recomendação de manuseio do paciente com SMD e escolha terapêutica está resumida nas Figuras 1,2,3

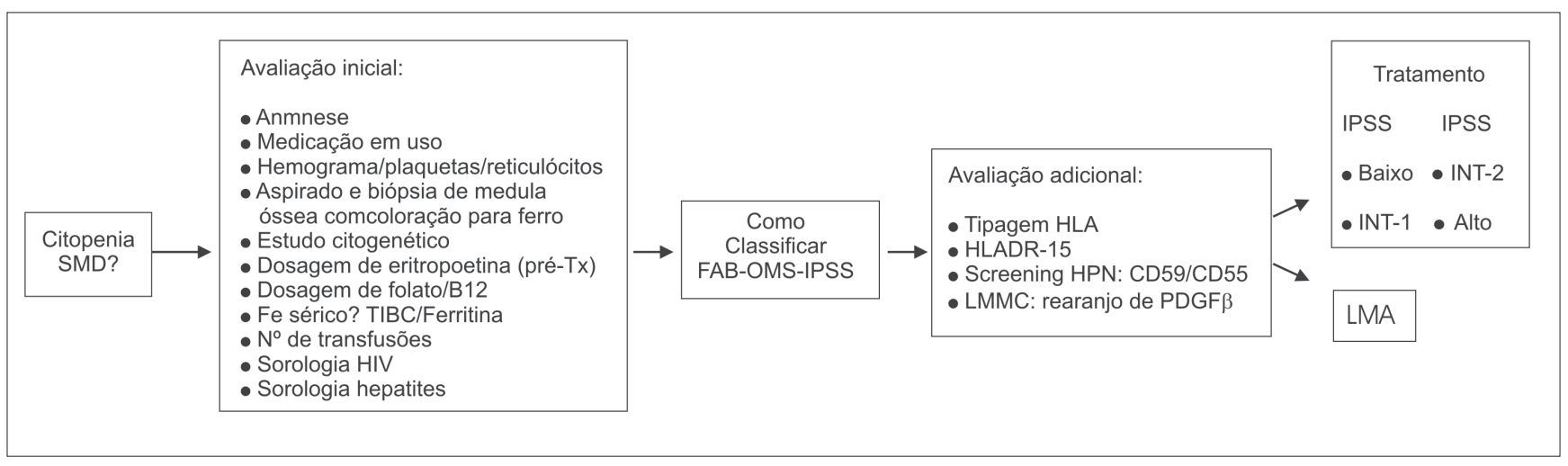

Figura 1

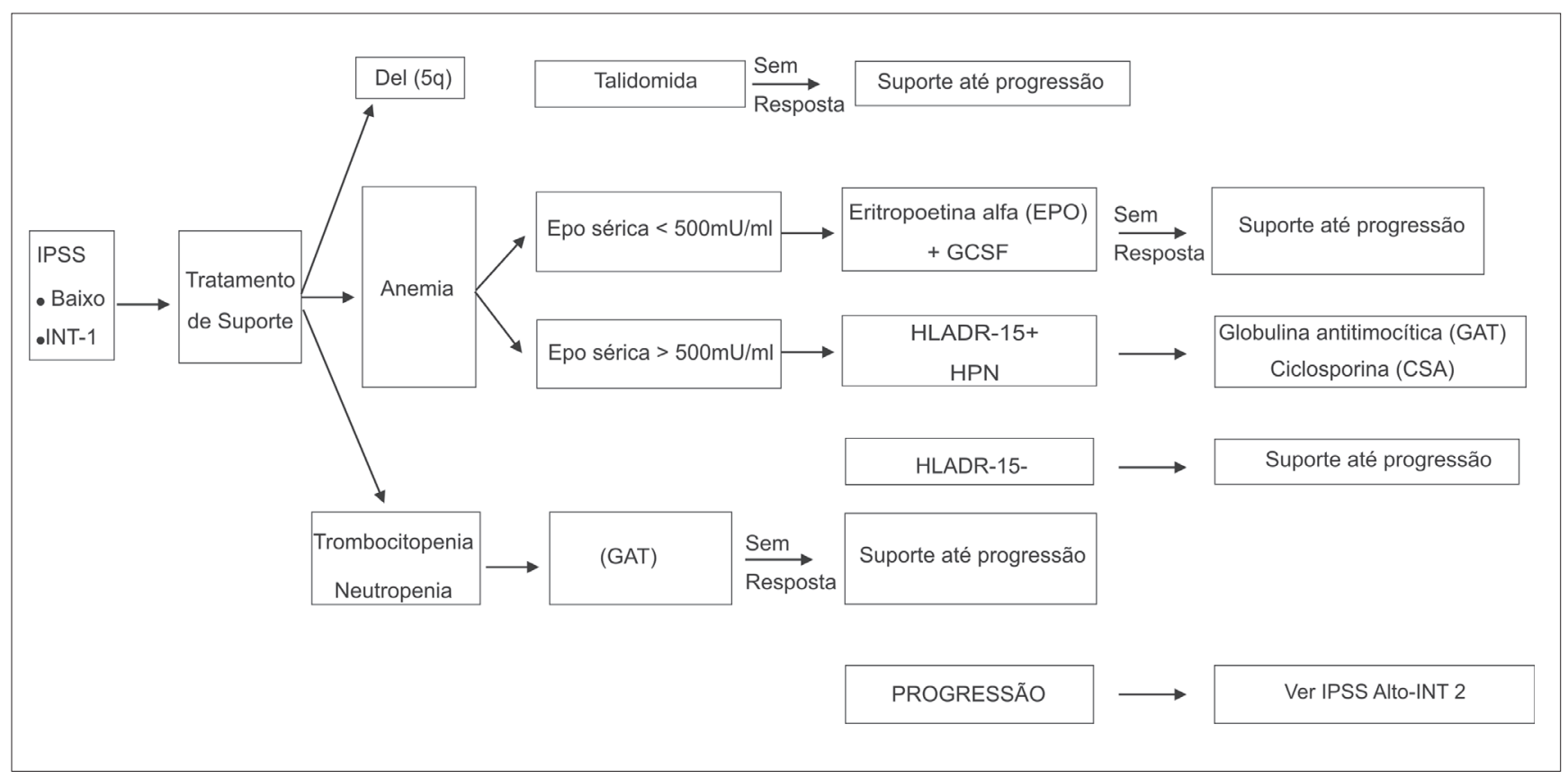

Figura 2 


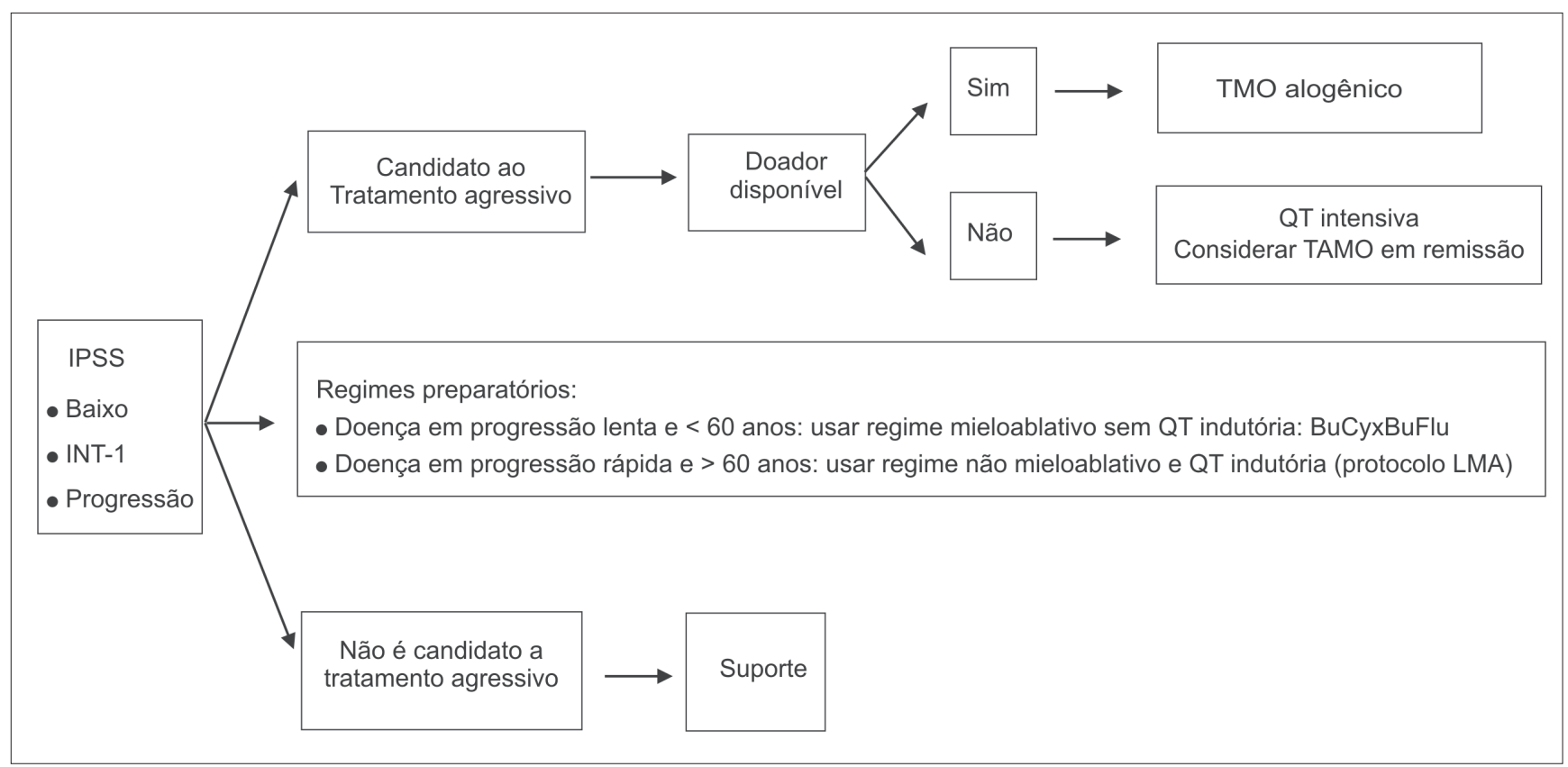

Figura 3

\section{Conclusão}

- O IPSS permite estabelecer uma estimativa de sobrevida dos pacientes.

- O transplante alogênico de células-tronco hematopoéticas permanece sendo a única opção terapêutica curativa para os pacientes com SMD.

- Os pacientes sem doador aparentado, o TCTH autólogo pode constituir uma alternativa terapêutica para os pacientes que alcançam remissão após quimioterapia de indução.

- Os RIST podem ser considerados para pacientes previamente selecionados.

- Com a melhora da terapia de suporte e o advento de novas drogas, como a 5-azacitidina e lenalidomida, os pacientes com SMD têm apresentado uma melhor qualidade de vida e maior sobrevida.

- Novas estratégias terapêuticas visando a prevenção das recidivas pós-transplante vêm sendo consideradas.

- A possibilidade de quelação de ferro deve ser considerada em pacientes de baixo risco que recebem transfusão de sangue regular.

\section{Abstract}

The myelodysplastic syndrome (MDS) encompasses a series of hematological conditions characterized by chronic cytopenias with abnormal cellular maturation. Based on the cytopenias, number of blast cells in bone marrow and cytogenetic abnormalities, MDS may be best classified by the International Prognostic Scoring System
(IPSS) in four groups: low risk, intermediate 1, intermediate 2 risks and high risk. A subset of patients can be cured following allogeneic hematopoietic stem cell transplantation (SCT). This therapy should be considered for under 60-year-old patients with an HLA-matched sibling donor. The decision in favor of this aggressive therapy depends upon a number of criteria including the IPSS score, risk of disease progression, risk of infection, and the overall health of the patient. Autologous HCT can be considered for those rare patients who are successfully induced into complete remission and do not have an HLA-matched donor. Non-myeloablative allogeneic HCT appears promising for patients with MDS who are not candidates for myeloablative allogeneic HCT. Early results are encouraging. Despite an increased relapsed rate, the treatment-related mortality is lower. Patients should be enrolled in well-designed clinical trials attempting to address the important issues of patient comorbidities, GVHD, and relapse risk. Rev. Bras. Hematol. Hemoter. 2010;32 (Supl. 1):66-70.

Key words: Hematopoietic cell transplant; myelodysplastic syndromes.

\section{Referências Bibliográficas}

1. Garcia-Manero G, Shan J, Faderl S, Cortes J, Ravandi F, Borthakur G, et al. A prognostic score for patients with lower risk myelodysplastic syndrome. Leukemia. 2008;22(3):538-43.

2. Cutler CS, Lee SJ, Greenberg P, Deeg HJ, Pérez WS, Anasetti C, et al. A decision analysis of allogeneic bone marrow transplantation for themyelodysplastic syndromes: delayed transplantation for lowrisk myelodysplasia isassociated with improved outcome. Blood. 2004;104(2):579-85

3. de Witte T, Suciu S, Brand R, Muus P, Kröger N. Autologous stem cell transplantation in myelodysplastic syndromes. Semin Hematol. 2007;44(4):274-7. 
4. De Witte T, Van Biezen A, Hermans J, Labopin M, Runde V, Or R, et al. Autologous bone marrow transplantation for patients with myelodysplastic syndrome (MDS) or acute myeloid leukemia following MDS. Chronic and Acute Leukemia Working Parties of the European Group for Blood and Marrow Transplantation. Blood. 1997;90(10):3853-7.

5. Woolfrey A, Gooley T, Malkki M, et al. Outcome of hematopoietic cell transplant from HLA-Matched Siblings Compared to a Volunteer Unrelated Donors Matched for HLA-A, B, C, DRB1, and DQB1 Alleles. Blood. 2007;110:170.

6. Castro-Malaspina H, Harris RE, Gajewski J, Ramsay N, Collins R, Dharan B, et al. Unrelated donor marrow transplantation for myelodysplastic syndromes: outcomeanalysis in 510 transplants facilitated by the National Marrow Donor Program. Blood. 2002 Mar 15;99(6):1943-51.

7. De Witte T, Hermans J, Vossen J, et al. Haematopoietic stem cell transplantation for patients with myelodysplastic syndromes and secondary acute myeloid leukaemias: a report on behalf of the Chronic Leukaemia Working Party of the European Group for Blood and Marrow Transplantation (EBMT). Br J Haematol 2000; 110:620-630

8. Laughlin MJ. Unrelated cord blood allogeneic stem cell transplantation for MDS. Leuk Lymphoma. 2006;47(4):569-70.

9. Deeg HJ, Shulman HM, Anderson JE, Bryant EM, Gooley TA, Slattery JT, et al. Allogeneic and syngeneic marrow transplantation for myelodysplastic syndrome in patients 55 to 66 years of age. Blood. 2000;95(4):1188-94.

10. Ho AY, Pagliuca A, Kenyon M, Parker JE, Mijovic A, Devereux $\mathrm{S}$, et al. Reduced-intensity allogeneic hematopoietic stem cell transplantation for myelodysplastic syndrome and acute myeloid leukemia with multilineage dysplasia using fludarabine, busulphan, and alemtuzumab (FBC) conditioning. Blood. 2004; 104(6):1616-23.

11. Spyridonidis A, Bertz H, Ihorst G, Grüllich C, Finke J. Hematopoietic cell transplantation from unrelated donors as an effective therapy for older patients ( $>$ or $=60$ years) with active myeloid malignancies. Blood. 2005;105(10):4147-8.
12. Locatelli F, Pession A, Bonetti F, Maserati E, Prete L, Pedrazzoli $\mathrm{P}$, et al. Busulfan, cyclophosphamide and melphalan as conditioning regimen for bone marrow transplantation in children with myelodysplastic syndromes. Leukemia. 1994;8(5):844-9.

13. Woolfrey AE, Gooley TA, Sievers EL, Milner LA, Andrews RG, Walters $\mathrm{M}$, et al. Bone marrow transplantation for children less than 2 years of age with acute myelogenous leukemia or myelodysplastic syndrome. Blood. 1998;92(10):3546-56.

14. Yusuf U, Frangoul HA, Gooley TA, Woolfrey AE, Carpenter PA, Andrews RG, et al. Allogeneic bone marrow transplantation in children with myelodysplastic syndrome or juvenile myelomonocytic leukemia: the Seattle experience. Bone Marrow Transplant. 2004;33(8):805-14.

15. Greenberg P, Bishop M, Deeg J et al. NCCN practice guidelines for Myelodysplastic Syndromes. http://www.cancernetwork.com/ journals/Oncology/NCCN9810.HTM.

Este artigo foi avaliado pelos coordenadores das Diretrizes do Transplante de Medula Óssea da Sociedade Brasileira de Transplante de Medula Óssea, Luis Fernando Bouzas, Prof. Julio Cesar Voltarelli e Nelson Hamerschlak, e publicado após avaliação e revisão do editor, Milton Artur Ruiz.

Conflito de interesse: sem conflito de interesse

Recebido: 09/11/2009

Aceito: 23/11/2009 